\title{
Bioelectrocatalysis and more
}

\section{Introduction}

Bioelectrocatalysis is a phenomenon widely explored by research community in various directions: biochemical assays for quantitative determination of different compounds of interest, food and pharmaceutical industry, cosmetics, production of detergents, wastewater treatment, etc. All these applications rely on the advantages of enzymatic and bacterial catalysis: specificity, selectivity, fast reaction rate and regulation capacity. The same advantages have been explored in the design of bioelectrochemical systems for energy conversion, biosensing applications and wastewater purification.

Bioelectrochemical systems also known, as biofuel cells are one of the most popular "non-conventional" fuel cells in the last 50 years and the most promising technology for conversion of renewable biomass to electrical power as well as wastewater treatment approach .$^{1,2}$ Biofuel cells can be divided into two main categories: enzymatic fuel cells (EFC) and microbial fuel cells (MFC). In both, oxidation of a given "fuel" occurs at the anode combined with a reduction of final electron acceptor at the cathode ${ }^{3}$ The potential difference between the two electrodes is the driving force of the processes, which leads to the transformation of chemical energy, stored in the "fuel" bonds, to electrical current ${ }^{4}$ Based on their operational principle these systems are classified as galvanic or fuel cells but what makes them "untraditional" is the nature of the catalysts used. In EFCs, the oxidation and reduction processes are catalyzed by the utilization of specific redox enzymes and in MFCs, the catalysts applied are microorganisms. The exploration of naturally occurring processes and phenomenon for the generation of electricity is the most beneficial feature of biofuel cells . They are biocompatible, cheap, selective, and effective at mild temperatures and neutral $\mathrm{pH}$. Therefore, biofuel cells can be a key technology toward the generation of clean and sustainable energy.

\section{Means and ways}

Many research efforts have been dedicated to the development and improvement of bioelectrochemical systems, specifically fundamental understanding of bioelectrocatalysis and internal and external electron transfer, materials selection, and design optimization. Based on the gained knowledge through the years, the generated power and current densities from laboratory prototypes of biofuel cells have increased significantly (from $0.05 \mathrm{~mA} / \mathrm{m}^{2}$ to $1000 \mathrm{~mA} / \mathrm{m}^{2}$ ) . ${ }^{6}$ However, after 100 years of research in this field, scientists and engineers have not yet realized dramatic improvements in energy densities and/or huge achievements as practical devices. There are two main reasons for that:

a. A bottom up approach needs to be explored - from fundamental understanding to engineering solutions;

b. The "one by one" approach commonly used in research should be replaced with multi-parameter approach that provides information on bioelectrochemical systems optimization based on understanding of how multiple parameters amplify each other and improve performance (Figure 1) .?
Volume 6 Issue 3 - 2017

\section{Sofia Babanova}

Chemical and Biological Engineering Department, University of New Mexico, USA

Correspondence: Sofia Babanova, Chemical and Biological Engineering Department, University of New Mexico, USA, Tel 5053588044 Email babanova@unm.edu

Received: September 01, 2017 | Published: October 03, 2017

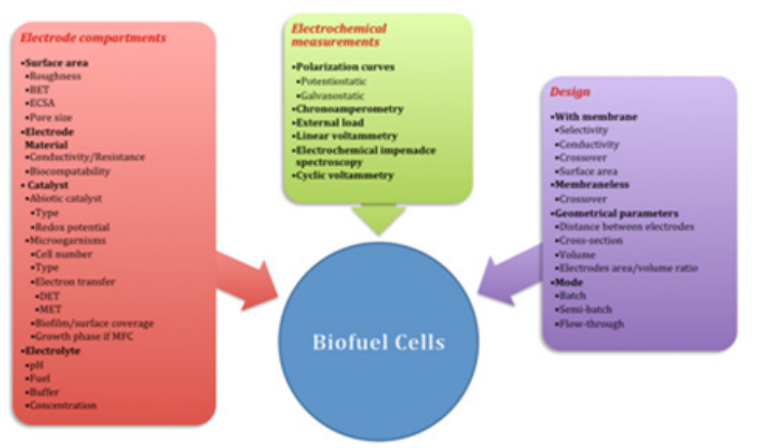

Figure I Factors determining bioelectrochemical systems performance. ${ }^{7}$

\section{Bottom up approach: Integration of biocatalysts with different solid supports for enhanced interfacial interactions}

The bottom up approach for bioelectrochemical systems starts with fundamental study and optimization of biocatalyst-support interactions as the main rate limiting step for enhanced electron transfer rate and improved performance. These include but are not limited to the development of various techniques for sufficient enzyme immobilization trough physical adsorption.$^{8}$ covalent attachment.$?$ physical entrapment and tethering of enzymes.$^{10}$ Mediators have been used to enhance electron transfer rate.$^{6,9,11}$ and carbon or mesoporous materials with diameters comparable to the size of the enzyme were explored for enhanced enzyme-electrode interactions.$^{12}$ The following approaches provide proper enzyme orientation: specific covalent attachment of enzymes to the electrode surface ${ }^{13,14}$ protein engineering.${ }^{15}$ and reconstitution of apo-enzymes directly on the electrode surface.$^{16}$

A new approach for improved enzyme-electrode interactions and ultimately for enhanced bacteria-electrode electron transfer has 
been developed ${ }^{17-19}$ This approach is based on the specific enzymesubstrate interactions, relying on key-lock principle that is the main advantage of enzymatic catalysis. Depending on the enzyme type two approaches were developed. The first approach uses modification of the electrode surface with enzyme's natural substrate or its functional analogue, which ultimately results in proper enzyme orientation and facilitated electron transfer (Figure 2). This hypothesis was applied for the orientation of two of the most famous oxygen-reducing enzymes, Bilirubin Oxidase and Laccase and its rationality was demonstrated in the development of oxygen reducing electrodes .${ }^{17,18}$ Further this hypothesis was transferred towards enhancement of electron transfer from "anodic" enzymes towards solid supports, where the enzyme terminal electron acceptor was utilized for electrode surface modification. This approach led to eight fold increase in the performance of an anode composed of pyrroloquinoline quinone (PQQ) dependent glucose dehydrogenase, very promising enzyme for the design of glucose sensors. ${ }^{20,21}$

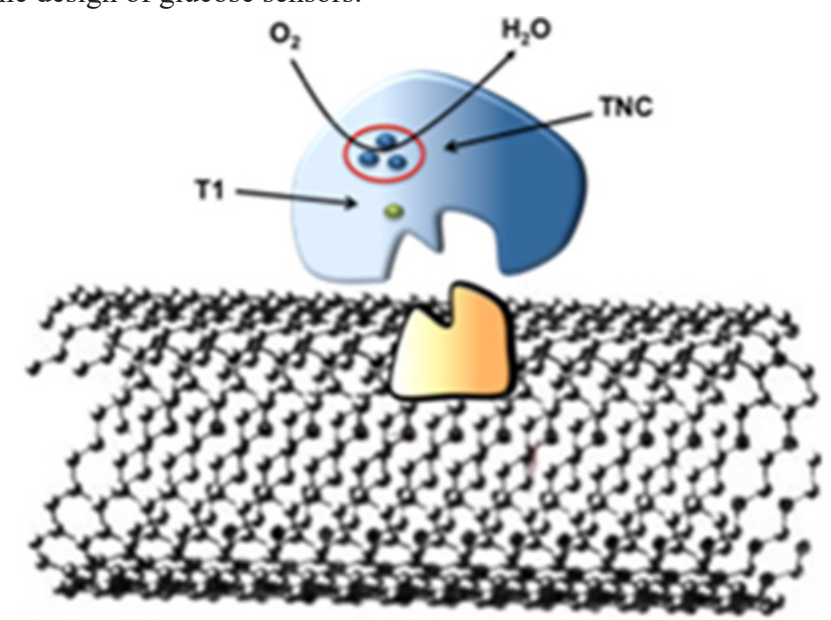

Figure $2 \mathrm{MCO}$ s orientation based on the key-lock principle. ${ }^{18}$

Currently research activities associated with the utilization of enzymes in bioelectrochemical systems are focused on understanding of the coenzyme location and structure as well as its participation in internal and external electron transfer. What is not known is: How these enzymes interact with their substrates or final electron acceptors in nature?; What structural changes accompany the enzyme immobilization and operation in biofuel cells?; What is the nature of enzyme-substrate recognition mechanism and interactions?; How this can be explored in the development of enzymatic electrodes with enhanced enzyme-electrode interactions?; Is the final electron acceptor diffusing into the enzyme molecule?; Is there a specific acceptor-binding pocket?; Is the same channel used for substrate and electron acceptor transportation, or there is another path for the acceptor to get close to the reduced cofactor?; What is the mechanism of the cofactor-electron acceptor interactions?; What happens when the enzyme contains more than one cofactor?; etc. These are some of the fundamental questions we need to find answers to and explore the knowledge in the development of practical systems based on enzymatic catalysis. The knowledge gained can be further transferred in the development of microbial electrodes and improvement of the bacteria-electrode communication.$^{22}$

Multi-parameter approach: Identify and isolate the factors having the biggest impact on the operational features of bioelectrochemical systems

Once the biological interfacial reactions are enhanced and are no longer the rate-limiting step in the operation of biofuel cells, understanding of the design parameters and their influence becomes a primary question.$^{23}$ Therefore, it is highly recommended to identify the key factors having the biggest impact on system operational features as well as system uncertainty. Unfortunately, due to the complexity of biofuel cells and mainly because of their interdisciplinary character, researchers consider the most important factors to be in the range of their knowledge and interests. Microbiologist and biochemists consider the biological component as determining BFCs operation. At the same time, engineers consider the design parameters as the most important once. Who is right?

To find the answer of this question slightly different approach needs to be explored. This approach is highly innovative in this field and it relies on statistical analysis and data interpretation. Through evaluation of the expanded uncertainty of microbial fuel cells, the variations in electrodes resistance and mostly difference in their electrochemical accessible surface area were identified as the main parameters causing irreproducibility of MFCs responses.$^{24,25}$ The idea of using statistical approaches for analysis of biofuel cells was further enlarged to include Design of Experiments, Principal Component Analysis (PCA) and Canonical Correspondence Analysis . 7,26-29 The applied statistical interpretation of the results from a significant amount of MFCs data showed that design parameters were the dominating factors when the necessity bacterial biofilm was developed. The same conclusion was made when the expanded uncertainties of the main operational characteristics were evaluated (Figure 3 ). ${ }^{28}$

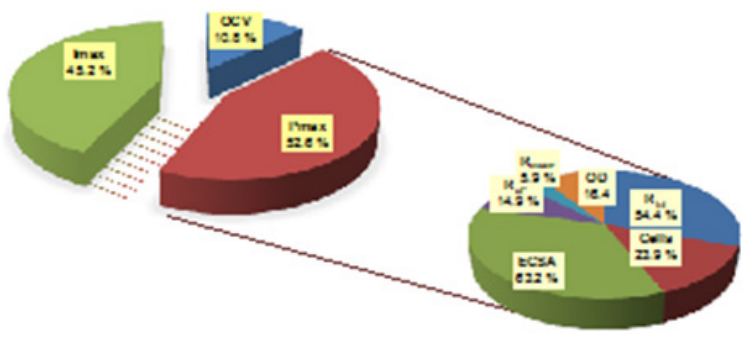

Figure 3 Uncertainties of the MFCs operational characteristics and several MFCs parameters. The uncertainty values in the pies are not normalized to $100 \%{ }^{28}$

a. Applying various statistical methods to evaluate the contribution of the different elements, factors and design on BFCs performance will give insights into the operation of these systems and the way of their optimization and commercialization. The following questions can find their answers:

b. What are the main factors influencing the BFCs performance (treatment rate, current and power densities)?

c. What is the ascendency of separate factors over the BFCs' operational characteristics?

d. Can we build a model combining the effects of these factors and describing the BFCs`operation?

e. Can we use this model to predict the final BFCs` output when one or more factors are varied?

f. How reproducible is BFCs performance?

g. From all factors, pertaining the BFCs, which ones are contributing mostly to the BFCs irreproducibility?

h. Which system parameter should be used to normalize the generated current and power? 
i. Are there statistically and scientifically validated points that should be used for data comparison?

Biofuel cell design parameters along with the operational conditions can be varied to emphasize the influence of a factor over the systems performance, not just in terms of electrochemical output but also towards other specific applications such as wastewater purification, quantitative determination of target compounds, etc. Identifying the main factors determining the biofuel cell performance, Principal Regression Analysis in combination with Path analysis can be used to build a mathematical model that can predict the final response of a system with a given parameters. This will dramatically decrease the experimental time and efforts for their optimization. Of course, to transfer a research idea into a technology, one main requirement should be fulfilled - reproducibility. Without reproducible performance, practical application cannot be possible.

Expanded uncertainty is a new term in the field of biofuel cells, which was recognized as necessary and introduced for the interpretation of results first from microbial fuel cells. ${ }^{24,25}$ As for conventional fuel cells, the commonly used electrochemical techniques for MFCs operational characteristics determination are polarization and power curves. The results from these polarization measurements are used for decision making if the studied fuel cell is appropriate for the intended purpose or not. Thus, uncertainty estimation of operational characteristics is required by the contemporary regulations for proper result presentation and comparison between results obtained in different laboratories under different experimental conditions. Study of the contributions of various uncertainty sources to the combined uncertainty allows to perform optimization of the experimental parameters having highest contribution to the uncertainty and, thus to obtain more reliable electrochemical system with reproducible output values. ${ }^{25}$ This is a question of crucial importance for the future practical application of biofuel cells especially when biosensors are developed.

\section{Conclusion}

The design and optimization of bio-electrodes and biofuel cells is not trivial. This technology bridges variety of research areas such as biotechnology, energy harvesting and generation, environmental science, micro- and nanostructured materials. Therefore, innovative and untraditional approaches need to be considered and combined.

\section{Acknowledgements}

Most the achievements referenced in this paper are a result of the work of two very important scientists, Prof. Plamen Atanassov and Dr. Orianna Bretscgher, to whom I am much obligated.

\section{Conflicts of Interest}

The authors declare no conflict of interest.

\section{References}

1. Meredith MT, Minteer SD Biofuel Cells: Enhanced Enzymatic Bioelectrocatalysis. Annu Rev Anal Chem. 2012;5(1):157-179.

2. Li, WW, Yu HQ, He Z Towards Sustainable Wastewater Treatment by Using Microbial Fuel Cells-Centered Technologies. Energy Environ Sci. 2014;7(3):911-924.

3. Barton SC, Gallaway J, Atanassov P Enzymatic Biofuel Cells for Implantable and Microscale Devices. Chem Rev 2004;104(10):4867-4886

4. Luckarift HR, Atanassov P, Johnson GR Enzymatic Fuel Cells: From Fundamentals to Applications. pp. 2014; 496.
5. Katz E, Mac Vittie K Implanted Biofuel Cells Operating in vivoMethods, Applications and Perspectives-Feature Article. Energy Environ Sci. 2013;6(10):2791.

6. Zebda A, Gondran C, Le Goff A et al. Mediatorless High-Power Glucose Biofuel Cells Based on Compressed Carbon Nanotube-Enzyme Electrodes. Nat Commun 2: pp. 2011;370.

7. Babanova S, Artyushkova K, Ulyanova Y et al. Design of Experiments and Principal Component Analysis as Approaches for Enhancing Performance of Gas-Diffusional Air-Breathing Bilirubin Oxidase Cathode. J Power Sources. 2014;245:389-397.

8. Flexer V, Durand F, Tsujimura S, Mano N Efficient Direct Electron Transfer of PQQ-Glucose Dehydrogenase on Carbon Cryogel Electrodes at Neutral pH. Anal Chem. 2011;83(14):5721-5727.

9. Flexer V, Mano N Wired Pyrroloquinoline Quinone Soluble Glucose Dehydrogenase Enzyme Electrodes Operating at Unprecedented Low Redox Potential. Anal Chem. 2014;86(5):2465-2473.

10. Strack G,Babanova S, Farrington KEetal. Enzyme-Modified Buckypaper for Bioelectrocatalysis. J Electrochem Soc. 2013;160(7):3178G-3182G.

11. Brocato S, Lau C, Atanassov P Mechanistic Study of Direct Electron Transfer in Bilirubin Oxidase. Electrochim Acta. 2012;61:44-49.

12. Ivnitski D, Atanassov $\mathrm{P}$, Apblett C Direct Bioelectrocatalysis of PQQ-Dependent Glucose Dehydrogenase. Electroanalysis. 2007;19(15):1562-1568.

13. Xu S, Minteer SD Enzymatic Biofuel Cell for Oxidation of Glucose to CO2. ACS Catal. 2012;2(1):91-94.

14. Göbel G, Schubart IW, Scherbahn V, Lisdat F Direct Electron Transfer of PQQ-Glucose Dehydrogenase at Modified Carbon Nanotubes Electrodes. Electrochem commun. 2011;13(11):1240-1243.

15. Okuda J, Sode K PQQ Glucose Dehydrogenase with Novel Electron Transfer Ability. Biochem Biophys Res Commun. 2004;314(3):793-797.

16. Sun D, Scott D, Cooney MJ, Liaw BY A Promising Reconstitution Platform for PQQ-Dependent Apo-Enzymes in Chitosan-Carbon Nanotube Matrices. Electrochem Solid State Lett. 2008;11(6):101B.

17. Lopez R J, Babanova S, Ulyanova Y et al. Improved Interfacial Electron Transfer in Modified Bilirubin Oxidase Biocathodes. Chem Electro Chem. 2014;1(1):241-248.

18. Ulyanova Y, Babanova S, Pinchon E et al. Effect of Enzymatic Orientation through the Use of Syringaldazine Molecules on Multiple Multi-Copper Oxidase Enzymes. Chem Phys. 2014;16(26):13367-13375.

19. Matanovic I, Babanova S, Chavez MS, Atanassov P Protein-Support Interactions for Rationally Designed Bilirubin Oxidase Based Cathode: A Computational Study. J Phys Chem B. 2016;120(15):3634-3641.

20. Babanova S, Matanovic I, Atanassov P Quinone-Modified Surfaces for Enhanced Enzyme-Electrode Interactions in PyrroloquinolineQuinone-Dependent Glucose Dehydrogenase Anodes. Chem Electro Chem. 2014;1(11):2017-2028.

21. Babanova S, Matanovic I, Chavez MS, Atanassov P Role of Quinones in Electron Transfer of PQQ-Glucose Dehydrogenase Anodes-Mediation or Orientation Effect. J Am Chem Soc. 2015;137(24):7754-7762.

22. Cornejo JA, LopezC, Babanova S et al. Surface Modification for Enhanced Biofilm Formation and Electron Transport in Shewanella Anodes. $J$ Electro Chem Soc. 2015;162(9): 597H-603H.

23. Bretschger O, Cheung ACM, Mansfeld F et al. Comparative Microbial Fuel Cell Evaluations of Shewanella Spp Electroanalysis 2010;22(7-8):883-894.

24. Roy JN, Babanova S, Garcia KE et al. Catalytic Biofilm Formation by Shewanella Oneidensis MR-1 and Anode Characterization by Expanded Uncertainty. Electrochim Acta. 2014;126:3-10. 
25. Babanova S, Hubenova Y, Mitov M et al. Uncertainties of YeastBased Biofuel Cell Operational Characteristics. Fuel Cells. 2011;11(6):824-837.

26. Santoro C, Babanova S, Artyushkova K et al. Influence of Anode Surface Chemistry on Microbial Fuel Cell Operation. Bioelectrochemistry. 2015;106(Pt A):141-149.

27. Santoro C, Guilizzoni M, Correa Baena JP et al. The Effects of Carbon Electrode Surface Properties on Bacteria Attachment and Start up Time of Microbial Fuel Cells. Carbon NY. 2014;67:128-139.
28. Babanova S, Bretschger O, Roy J, Cheung A, Artyushkova K, et al. (Innovative Statistical Interpretation of Shewanella Oneidensis Microbial Fuel Cells Data. Phys Chem Chem Phys. 2014;16(19):8956-8969.

29. Ishii S, Suzuki S, Norden Krichmar TM et al. Microbial Population and Functional Dynamics Associated with Surface Potential and Carbon Metabolism. ISME J. 2014;8(5):963-978. 